$$
\text { "tmcs-dikovic" — 2009/11/1 — 16:54 — page } 241 \text { — \#1 }
$$

\title{
Examining continuity/discontinuity of a function by using GeoGebra
}

\author{
LJUBica Dikovic
}

\begin{abstract}
The possibility to visualize the things with the help of today's dynamic software (GeoGebra being one of them), enables the students to see and explore mathematical relations and concepts that were difficult to be presented in the past, prior to the state-of-the-art technologies. In methodological sense, the contribution of this paper lies in the presentation of a set of visualizations designed to help students better understand and explore the basic calculus concepts such as continuity at a point, to examine discontinuity at a point, to display discontinuities and the relations between continuity and differentiability of single variable functions. In technical sense, this paper presents creative GeoGebra applets which offer new possibilities that could be of a vital importance for the future development of e-learning of College mathematics.
\end{abstract}

Key words and phrases: GeoGebra, continuity, discontinuity, college mathematics, calculus.

ZDM Subject Classification: R20, I40, D40, N80.

\section{Integration of technology in the traditional teaching of mathematics}

The modern vision of education has been relying on the application of new communication and computer technologies with the aim of grasping new levels of creativity and knowledge in the context of the investigation.

ICTs and 'Computer Aided Teaching' have become an important part of life today, and are widely used to improve teaching and learning techniques. The main forms in teaching mathematics with computers are:

Copyright (C) 2009 by University of Debrecen 


$$
\text { "tmcs-dikovic" — 2009/11/1 — 16:54 — page } 242 \text { — \#2 }
$$

- Web teaching and learning systems.

Using ICTs many Universities have established virtual education and distance learning systems in the field of mathematics, with all new possibilities for students.

- Mathematics software packages.

Many mathematics software packages have been developed, which have very powerful, numerous functions, such as: Instantaneous numerical and symbolic calculations; Data collecting, analysis, exploration, and visualization; Modeling, simulation, and prototyping; Presentation graphics and animation in 2D and 3D; Application development.

The goal is to use technologies which need to provide an environment for active exploration of mathematical structures through multiple representations, or to show students some aspects of the mathematics that are not possible with pen and paper. The students can concentrate on ideas; additionally computers can stimulate the students' geometric intuition through interesting visualizations in $2 \mathrm{D}$ or $3 \mathrm{D}$, or make it possible to ask questions involving theoretical issues that are arithmetically too complicated in the traditional way (Dikovic, 2007).

Research suggests that despite the numerous benefits of using technology in mathematics education, the process of embedding technology in classrooms is slow and complex (Hohenwarter, M., \& Lavicza, Z., 2007). It is true that the extent of ICT use in the classroom is dependant on the individual teacher.

NCTM (2000) states that:

The effective use of technology in the mathematics classroom depends on the teacher. Technology is not a panacea. As with any teaching tool, it can be used well or poorly. Teachers should use technology to enhance their students' learning opportunities by selecting or creating mathematical tasks that take advantage of what technology can do efficiently and well - graphing, visualizing, and computing (p. 25).

Although students often can work independently of the teacher when using computers and appropriate software, new technology never will be able to replace teachers, since they play vital roles in technology-rich classroom settings. Thereby, technology potentially opens up new observation possibilities for teachers, allowing them to focus on students' investigations and thinking strategies while solving mathematical problems (p. 25).

The use of computer packages results in more independent productive student activity. Computer lessons require less class teaching and more partner and 


$$
\text { "tmcs-dikovic" — 2009/11/1 — 16:54 — page } 243 \text { — \#3 }
$$

individual work as well as less note taking and more production. For solving problems and making concepts it is a great advantage that the students can approach situations numerically, graphically and symbolically (Wurnig, 2008).

The concepts of differentiation and integration are fundamental to understanding many mathematical connections with other natural and social sciences, and with business. To fully understand these mathematical concepts, it is necessary that students have experience through passive observances of the teacher's work and through own, active work with multiple and interconnecting representations (verbal, symbolic, numerical and graphic) of the concepts (Blubaugh, 2004). In any case, the proper instruction tool must be selected and used in a way so that technology does not become a barrier between the student and the concept(s) being addressed.

The visualization that is possible with today's dynamic software enables the student to see and explore mathematical relations and concepts that were difficult to "show" in past prior to technology. The most meta-analysis of research in the area of instructional technology in education show that students who use technology in their learning had positive gains in learning outcomes over students who learned without technology (Waxman, Connell, Gray, 2002).

\section{Discovery Learning}

Cognitive tools are defined as instruments included in a learning environment allowing learners to make cognitive processes, like discovery skills, and their results explicit. Cognitive tools therefore can play a supportive role in discovering a domain. Important characteristics of cognitive tools are: (Joolingen, 1999)

- A design theory for cognitive tools is necessary to be able to offer genuine support for discovery learning.

- Cognitive tools have intended and unintended effect on discovery learning processes. They influence the way discovery processes are carried out and the learners' freedom.

- The integration of cognitive tools in a simulation environment.

In this paper some cognitive tools will be presented and discussed, as they appear in the GeoGebra environment for simulation-based discovery learning.

Discovery learning is a type of learning where learners construct their own knowledge by experimenting with a domain, and inferring rules from the results of these experiments. The basic idea of this kind of learning is that because

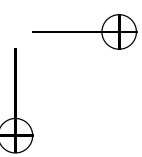




$$
\text { "tmcs-dikovic" — 2009/11/1 — 16:54 — page } 244 \text { — \#4 }
$$

learners can design their own experiments in the domain and infer the rules of the domain themselves they are actually constructing their knowledge. Because of these constructive activities, it is assumed they will understand the domain at a higher level than when the necessary information is just presented by a teacher. Discovery Learning is a method of inquiry-based instruction; discovery learning believes that it is best for learners to discover facts and relationships for themselves (Bruner). [14]

Proponents of this theory believe that discovery learning has many advantages, including: encourages active engagement, promotes motivation, and promotes autonomy, responsibility, independence, the development of creativity and problem solving skills and a tailored learning experience.

We can define cognitive tools as being instruments that are designed for supporting cognitive processes and thereby extending the limits of the human cognitive capacities. In principle anything can be a cognitive tool, for instance a sheet of paper and a pencil can be a cognitive tool to support the cognitive process of remembering items, extending the limited capacity of working memory.

When applied to learning, cognitive tools can be seen as supporting learning processes. Learning processes are the basic entities of describing the activities a learner needs to do in order to increase his or her understanding of a certain domain. This can be to remember something, to practice a procedure, to solve a problem, to set a hypothesis or some other process. Learning processes, when carried out properly, contribute to the construction of knowledge by the learner. However, learning processes may be quite difficult for a learner to perform, for instance because the process is very complex, not understood or because several learning processes need to be carried out at the same time.

\section{Geogebra?}

At the moment, GeoGebra (from Geometry and Algebra) is one of the most innovative, open-code math software (GNU General Public License) which can be freely downloaded from www.geogebra.org.

GeoGebra works on a wide spectrum of operating system platforms which have Java virtual machine installed on.

Markus Hohenwarter created free, open-source dynamic mathematics software GeoGebra, which is used for both teaching and learning mathematics from middle school through college to the University level (see Hohenwarter \& Preiner, 


$$
\text { "tmcs-dikovic" — 2009/11/1 — 16:54 — page 245 — \#5 }
$$

2007). GeoGebra offers geometry, algebra and calculus features in a fully connected, compacted and easy-to-use software environment. In other words, this tool extends the concepts of dynamic geometry to the fields of algebra and mathematical analysis.

Designed specifically for educational purposes, GeoGebra can help students grasp experimental, problem-oriented and research-oriented learning of mathematics, both in the classroom and at home.

Students can simultaneously use a computer algebra system and an interactive geometric system; by doing this, they can increase their cognitive abilities in the best way.

GeoGebra is dynamic geometry software that supports constructions with points, lines and all conic sections. It also provides typical features for a Computer Algebra System such as finding important points of functions (roots, local extrema and inflection points of functions), direct input of equations and coordinates, finding derivates and integrals of the entered functions. That is the reason why GeoGebra is a good choice for multiple presentations of mathematical objects. [6]

The basic idea of GeoGebra's interface is to provide two presentations of each mathematical object in its algebra and graphics windows. If you change an object in one of these windows, its presentation in the other one will be immediately updated.

To use a dynamic worksheet, students don't need GeoGebra installed on their computers. The only requirements are a Web browser and Java.

Also, trained teachers can convert those dynamic, virtual worksheets into interactive exercises with GeoGebra's JavaScript interface and through setting applet parameters. This provides an incredible flexibility; it also allows the creation of the exercises with automatic feedback or the exercises that are randomly generated.

For teachers, GeoGebra offers a powerful opportunity to create interactive, stimulating, online learning environments, the fact that has encouraged many of them to share free materials on the Internet.

Computer algebra systems (such as Mathematica, Maple, and so on, e.g.) and dynamic geometry software (such as Geometer's Sketchpad, Cabri Geometry, and so on, e.g.) are powerful technological tools for teaching mathematics. Numerous research results suggest that these software packages can be used to encourage discovery, experimentation and visualization in traditional teaching of mathematics. 


$$
\text { "tmcs-dikovic" — 2009/11/1 — 16:54 — page } 246 \text { — \#6 }
$$

However, researches suggest that, for the majority of teachers, the main problem is how to provide the technology necessary for the successful integration of technology into teaching (Ruthven \& Hennessy, 2004).

So, the suggested solution for applying technology in the college math teaching is the software packet GeoGebra.

The advantages of using GeoGebra are:

- In comparison to a graph calculator, GeoGebra is more user-friendly. GeoGebra offers easy-to-use interface, multilingual menus, commands and help.

- GeoGebra encourages students' projects in mathematics, multiple presentations and experimental and guided discovery learning.

- Students can personalize their own creations through the adaptation of interface (e.g. font size, language, quality of graphics, color, coordinates, line thickness, line style and other features).

- GeoGebra was created to help students gain a better understanding of mathematics. Students can manipulate variables easily by simply dragging "free" objects around the plane of drawing, or by using sliders. Students can generate changes using a technique of manipulating free objects, and then they can learn how the dependent objects will be affected. In this way, students have the opportunity to solve problems by investigating mathematical relations dynamically.

- Cooperative learning is the right context for a mathematics course (Dubinsky \& Schwingendorf, 2004). Lecturing should be replaced by a task-oriented interactive classroom. The primary role of teaching is not to lecture, explain, or otherwise attempt to "transfer" mathematical knowledge, but to create situations for students that will foster their making the necessary mental constructions. In that sense, GeoGebra provides a good opportunity for cooperative learning, i.e. cooperative problem solving in small groups, or whole class interactive teaching, or individual/group student presentations.

- The algebra input allows the user to generate new objects or to modify those already existing, by the command line. The worksheet files can easily be published as Web pages.

- GeoGebra stimulates teachers to use and assess technology in: visualization of mathematics; investigations in mathematics; interactive mathematics classes on site or at a distance; mathematics and its applications, etc.

The deficiencies of using GeoGebra are: 


$$
\text { "tmcs-dikovic" — 2009/11/1 — 16:54 — page } 247 \text { — \# }
$$

- Students without previous programming experience will hardly enter algebraic commands in the input box. Although the basic commands are not difficult to learn, students may feel embarrassed or quite at a loss of what to do.

- Some methodological approaches (e.g. independent exploring and experimenting) can not be appropriate for many students.

- In a technical sense, GeoGebra does not have an in-built support for animation. So, including the modules for animating in GeoGebra should become an important technical element for future versions.

- Future extensions of the software GeoGebra will surely include more symbolic features of computer algebra systems which will further increase possible complex applications in the mathematical analyses, and 3D extensions.

- Limited research on the impact of GeoGebra on teaching and learning of mathematics.

GeoGebra has been rapidly gaining popularity among teachers and researchers around the world, because it is easy-to-use dynamic mathematics software that combines many aspects of different mathematical packages. In addition, because of its open-source nature, an extensive user community has developed around it.

\section{Using GeoGebra for examining continuity/discontinuity of a function}

Descartes gave the first definition of function continuity saying that a function was continuous at an interval if its graph could be drawn without lifting a pencil off the paper. The formal definition of continuity started with defining continuity at a point, and than the definition was spread to the continuity on an interval. Although, at first, it seams that there is no connection between the formal and the informal definition of continuity, our goal is to try to make this connection obvious to our students by using a certain number of GeoGebra applets. In addition, we must point out that, with GeoGebra applets, the students get an algebraic and a geometric solution of the problem at the same time.

In general, we say that the function $y=f(x)$ is continuous at some point $x=a$ of its domain if, and only if, the following three conditions are satisfied:

$f(x)$ is defined at point $x=a$ i.e. $f(a)$ exists, $\lim _{x \rightarrow a} f(x)$ exists (i.e. is finite), and $\lim _{x \rightarrow a} f(x)=f(a)$. 
It is supposed that students hold pre-knowledge like: Function $f$ is said to be continuous on an interval, if $f$ is continuous at each point $x$ in that interval; Sum (difference, product) of continuous functions is continuous, or quotient of continuous functions is continuous at all points $x$ where the denominator is not zero, or functional composition of continuous functions is continuous at all points $x$ where the composition is properly defined; Any polynomial function (or $e^{x}$, $\sin x, \cos x)$ is continuous for all values of $x$, etc.

How does continuity affect limits? What is Cauchy. i.e. epsilon-delta definition of continuity?

Intuitively, a continuous function is a function for which, changes in some small neighborhood around input points, result in small changes in the output points. Because the symbolic relationships between limits and continuity are quite abstract and complicated, it would be useful to start with the applet designed to help users understand continuity in terms of limits, i.e. the epsilon/delta definition of continuity at a point $x_{0}$ (see [20] for function $f(x)=x^{n}$, with possibility to select $n, x_{0}$ and value for $\varepsilon$ ). This applet helps to understand the answer on the following question:

For any number $\varepsilon>0$, however small, can you find some number $\delta>0$ such that whenever $\left|x-x_{0}\right|<\delta$ then $\left|f(x)-f\left(x_{0}\right)\right|<\varepsilon$ (for all $x$ in the domain of $f$ )?

In order to develop an intuitive understanding and discover of continuity, next we can consider the following questions: What does it mean for a function to be continuous? What do continuous functions look like?

ExAMPLE 1. Let $f(x)= \begin{cases}\frac{x-6}{x-3}, & x<0 \\ 2, & x=0 . \\ \sqrt{4+x^{2}}, & x>0\end{cases}$

See if the given function is continuous at the point $x=0$.

With the help of GeoGebra, an appropriate applet has been created, in which the student gets the following algebraic explanation together with a graphic one (see Figure 1). The teacher can analyze and explain every segment of the given algebraic explanation:

The function $f(x)$ is defined at $x=0$, since $f(0)=2$. The left-hand limit of the function $\lim _{x \rightarrow 0_{-}} f(x)=\lim _{x \rightarrow 0_{-}} \frac{x-6}{x-3}=2$, and the right-hand limit of the function $\lim _{x \rightarrow 0_{+}} f(x)=\lim _{x \rightarrow 0_{+}} \sqrt{4+x^{2}}=2$ are calculated. As the left and the right limit values are equal, the conclusion is that there is a limit value of the 
function $\lim _{x \rightarrow 0} f(x)=2$. Since the condition that $\lim _{x \rightarrow 0} f(x)=2=f(0)=2$ is fulfilled, the function $f(x)$ is continuous at $x=0$.

On the graphic presentation, the applet also shows that $f(x)$ is continuous at $x=0$. By moving the "movable" point $A$ along the graph, and especially by approaching the point $x=0$ from the left and from the right, the student can confirm that the function has no so-called "jumps" when it passes through the point $x=0$.

Graphically, applet shows that $f(x)$ is continuous at $x=0$. Student can move the point $A$, approaches to point at $x=0$ and can verify that function has not "jumped" at $x=0$.

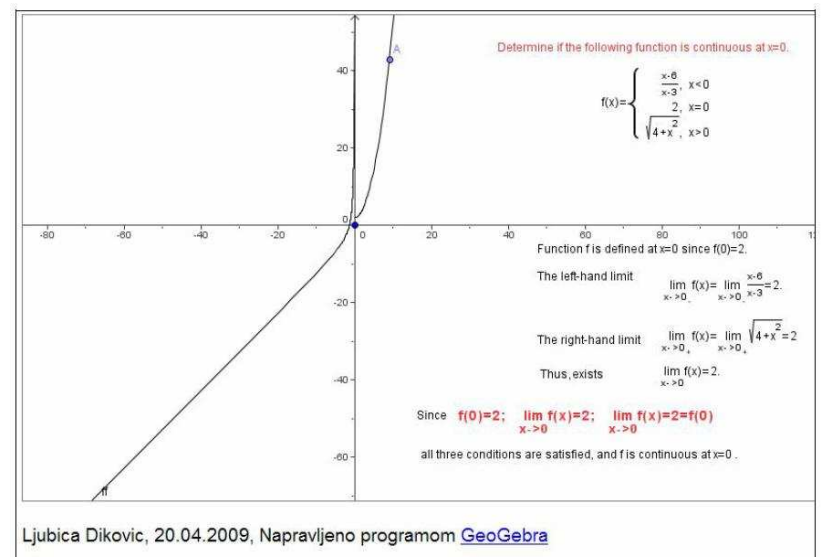

Figure 1. The example of the function which is continuous at the point $x=0$

If a function is not continuous at a point of its domain, it is said that it has a discontinuity at that point. Why is not a function continuous? What do the graphs of discontinuous functions look like? What types of discontinuities exist? Can a discontinuity be removed?

Indirectly, the definition of continuity at a point may make more sense if you see it applied to the functions with discontinuities.

Also, the presentation of discontinuity at a point and the development of geometric understanding and recognition of the graphs of discontinuous functions may be successfully illustrated by appropriate examples done in GeoGebra. 


$$
\text { "tmcs-dikovic" — 2009/11/1 — 16:54 - page } 250 \text { — \#10 }
$$

EXAMPLE 2. Examine the continuity of the $f(x)=\operatorname{sgn}(x)= \begin{cases}-1, & x<0 \\ 0, & x=0 \\ 1, & x>0\end{cases}$

It would be desirable for the algebraic analysis to precede the graphic presentation in the following example:

It is easy to notice that the limit value of the function, as $x$ approaches 0 from the right is 1 ; while the limit value of $f(x)$ as $x$ approaches 0 from the left is -1 . So, as the left- and right-hand limit values exist but are not equal, the point $x=0$ is the point of discontinuity for the given function.

Graphically, this discontinuity is presented as a "jump" at the point $x=0$ (see Figure 2). In this case, we can use GeoGebra applet to, graphically, make the "fine" comparisons of the function values in the small domain around the point $x=0$.

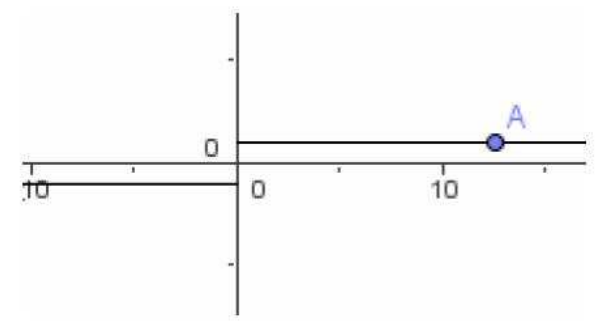

Figure 2. The example of the function with a "jump" at the point $x=0$.

ExAmple 3. Let $f(x)=\left\{\begin{array}{ll}3 x-4, & x \neq 1 \\ 3, & x=1\end{array}\right.$.

Examine the continuity of the function at the point $x=1$.

The appropriate GeoGebra applet offers the following algebraic explanation:

The function $f(x)$ is defined at the point $x=1$, that is $f(1)=3$. The limit value $\lim _{x \rightarrow 1} f(x)=\lim _{x \rightarrow 1} 3 x-4=-1$ is calculated. Given that the $\lim _{x \rightarrow 1} f(x)=-1 \neq f(1)=3$, the condition of continuity is not fulfilled, so the function $f(x)$ has a discontinuity at $x=1$.

Graphically, this discontinuity is presented as a "jump" at the point with coordinates $(1,3)$ (see Figure 3). 

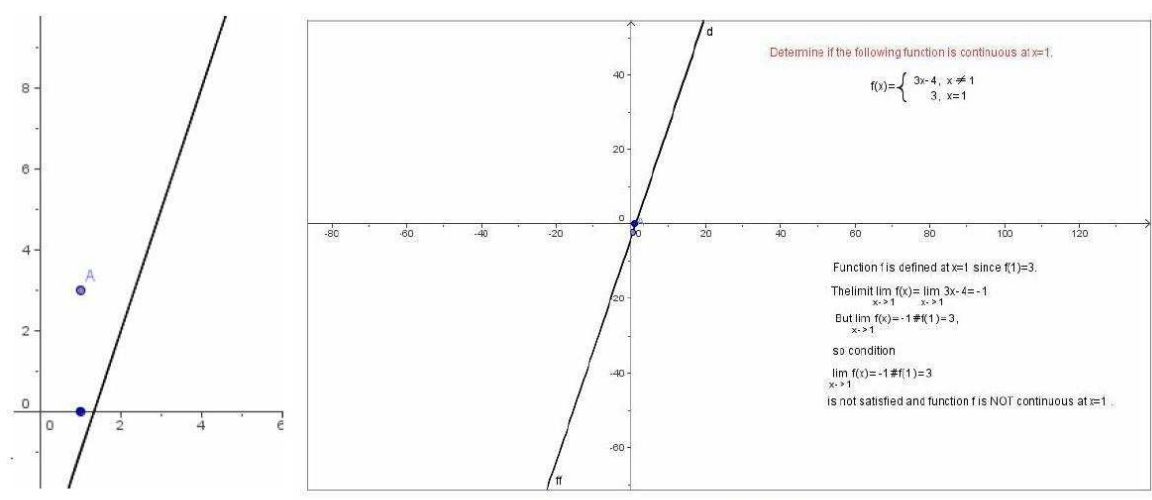

Ljubica Dikovic, 08.01.2009, Napravljeno programom GeoGebra

Figure 3. The example of the function with discontinuity at the point $x=1$

Example 4. Examine the function $f(x)=\left\{\begin{array}{ll}x^{2}, & x<1 \\ -2, & x=1 . \\ 2-x, & x>1\end{array}\right.$. Which type of discontinuity appears at the point $x=1$ ? Examine the discontinuity at the point $x=1$ by using GeoGebra "Include the Trace" option (see Figure 4).

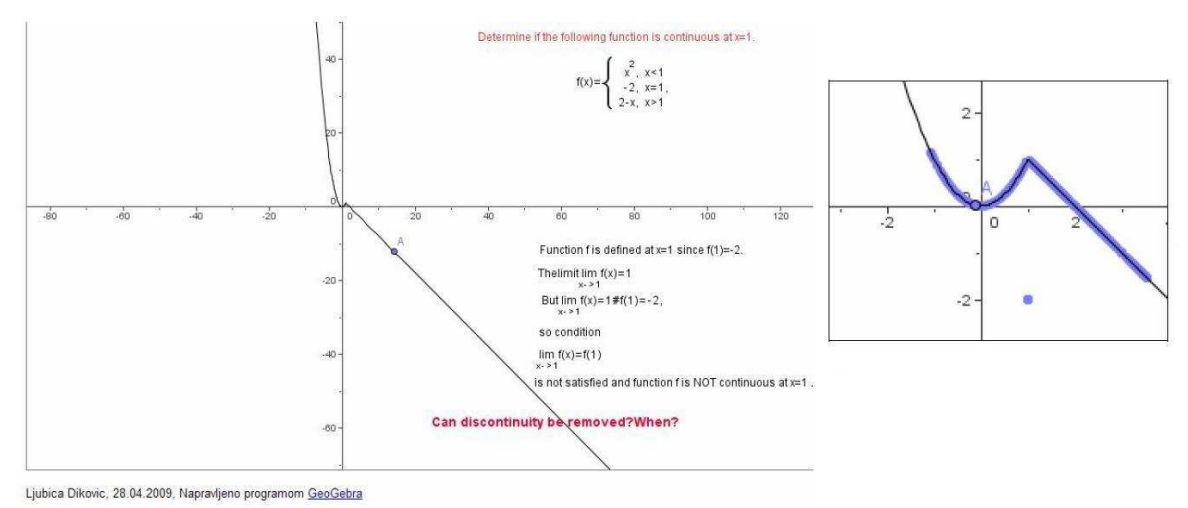

Figure 4. The example of the function with the removable discontinuity at the point $x=1$.

The appropriate GeoGebra applet offers the following algebraic explanation; 
It is easily checked that the left- and the right-hand limit values, as $x$ approaches 1 , are equal and are 1 . As there is a limit value as $x$ approaches 1 , the function has a removable discontinuity at $x=1$. If we define that $f(1)=1$ instead of $f(1)=-2$, then the function would become continuous on the whole real line. Graphically, the discontinuity is seen as a "jump" at the point with coordinates $(1,-2)$ (see Figure 4). With the help of GeoGebra "Include the Trace" option, the students can clearly notice, on the graph, the discontinuity of the function at $x=1$.

ExAmple 5. Examine the function $f(x)=\left\{\begin{array}{ll}\sin (1 / x), & x \neq 0 \\ x, & x=0\end{array}\right.$. Which type of discontinuity appears at $x=0$ ? Confirm your assumptions by zooming on the applet the epsilon interval around the point $x=0$ (see Figure 5).
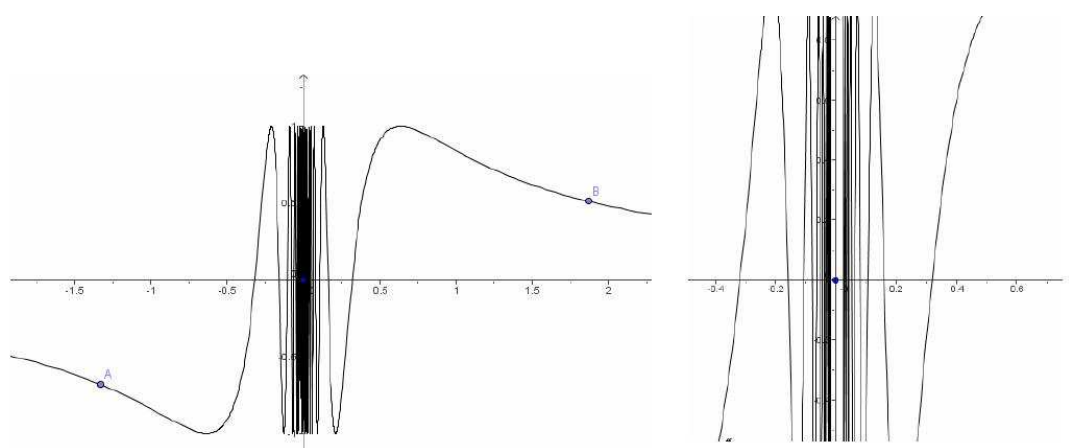

Figure 5. The example of the function that has an irremovable discontinuity at $x=0$.

Prior to the graphical, the students should be offered an algebraic explanation.

The sequence $x_{n}=\frac{1}{2 n \pi} \stackrel{n \rightarrow \infty}{\longrightarrow} 0_{+}$and $(\forall n) \quad f\left(x_{n}\right)=\sin (2 n \pi)=0$. The sequence $x_{n}=\frac{2}{(2 n+1) \pi} \stackrel{n \rightarrow \infty \pi}{\longrightarrow} 0_{+}$but $(\forall n)-1 \leq f\left(x_{n}\right)=\sin \left((2 n+1) \frac{\pi}{2}\right) \leq 1$.

Therefore, the limit value of $f(x)$ when $x$ approaches zero from the right does not exist. Because $f(x)$ is an odd function, the same arguments can be used for examining the left-hand limit values of the function. Thus, the function has a discontinuity of other type at the point $x=0$. The discontinuity is represented as a split in the graph at. In this more complicated case, we can use GeoGebra applet 


$$
\text { "tmcs-dikovic" — 2009/11/1 — 16:54 — page } 253 \text { — \#13 }
$$

(see Figure 5) and then compare graphically the function values in $\varepsilon$-domain of the point $x=0$ (by using the zoom option of GeoGebra).

EXAMPLE 6 . Which type of discontinuity the function $f(x)=x \sin (1 / x)$ has at the point $x=0$ ? What kind of discontinuity does the function $f(x)=$ $x \sin (1 / x)$ have at $x=0$ ?

Since $|x \sin (1 / x)|<|x|$, we can conclude that $f(x)$ tends to 0 when $x$ approaches zero from both sides. This means that the function has a removable discontinuity at $x=0$; or, if we define that $f(0)=0$, then the given $f(x)$ becomes continuous.

What is the connection between continuity and differentiability?

All differentiable functions are continuous, but that does not necessarily work the other way round: all continuous functions are not differentiable. My work experience shows that it is very difficult for the students to notice the connections between limit values, continuity and differentiability. The connection between continuity and differentiability can be noticed, with the help of applets, on the following examples:

ExAmple 7. Let $f(x)=\left\{\begin{array}{ll}x \sin (1 / x), & x \neq 0 \\ 0, & x=0\end{array}\right.$.

Confirm that the given function is continuous, but not differentiable at $x=0$.

It is usual for a teacher to analyze the algebraic solution of this standard example in a traditional way, but it would be desirable to offer a graphical visualization of the solution of the problem through GeoGebra. (see Figure 6 and Figure 7).

The next applet further develops a geometric understanding of the graphs of continuous functions. The student can easily see that the function is continuous everywhere, by simply "sliding" the point $A$ along the curve without break.

At the same time, the applet shows that $f(x)$ is not differentiable at the critical point $(0,0)$. We notice the secant lines through points $(0, f(0))$ and $(h, f(h))$, which oscillate between lines $y=x$ and $y=-x$. If were differentiable, these secant lines would be approximated by the tangent line in case when $h \rightarrow 0$, which did not happen. The student can watch the tangent lines by moving the point $A$ along the curve, can approach the point $x=0$ from the left and from the right, and then visually notice that there is no tangent line at the point $(0,0)$. 
Parallel with geometrical, the applet in question offers the algebraic proof that the function is continuous, but not differentiable at the point $x=0$.

On the next applet, the students can analyze the situations in which $x$ is replaced by $x^{2}$, that is, they can confirm that the result will be a function which is continuous and differentiable at the point $x=0$.

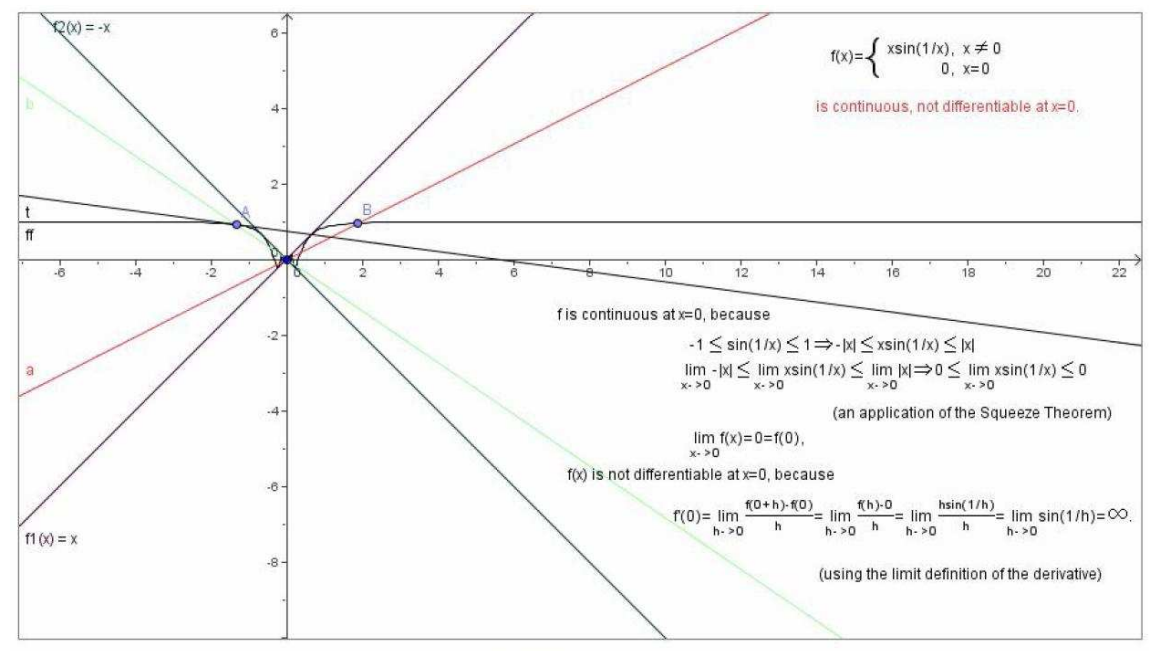

Ljubica Dikovic, 08.01.2009, Napravljeno programom GeoGebra

Figure 6. The function is continuous, but not differentiable at $x=0$
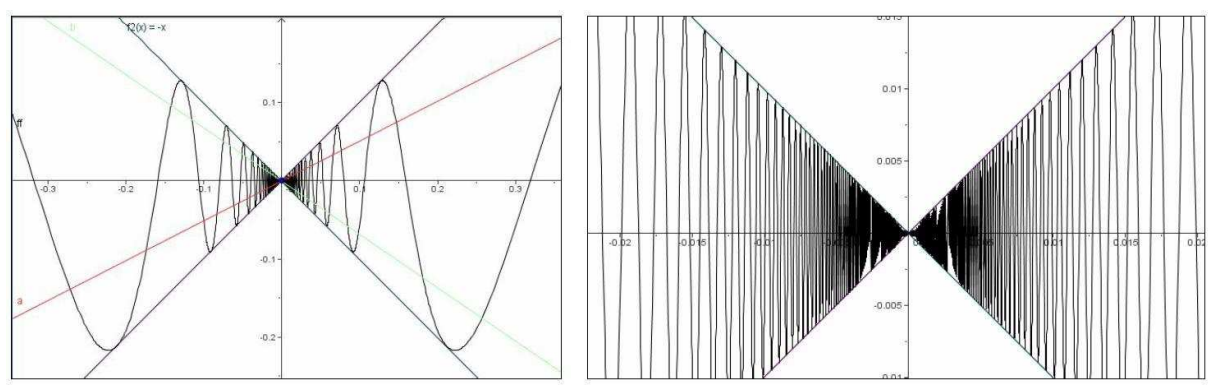

Figure 7. Zoom in (drawing pad) for Figure 6 


$$
\text { "tmcs-dikovic" — 2009/11/1 — 16:54 - page } 255 \text { — \#15 }
$$

ExAmple 8. Let $f(x)=\left\{\begin{array}{ll}x^{2} \sin (1 / x), & x \neq 0 \\ 0, & x=0\end{array}\right.$.

Prove that the function is continuous and differentiable at the point $x=0$.

This applet also develops a geometrical connection between continuity and differentiability of a function, offering, also, an appropriate algebraic solution to be noticed and analyzed. (See Figure 8).

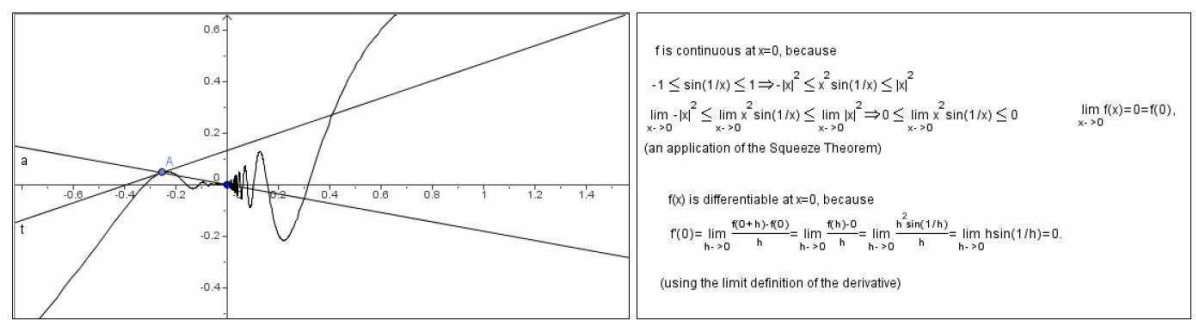

Figure 8. The example of a function which is continuous and differentiable at the point $x=0$.

\section{Conclusion}

In this paper, I highlighted some possibilities and examples of how GeoGebra can be used in classrooms to explore the basic calculus concepts such as continuity at a point, investigating discontinuity at a point, displaying discontinuities, learning how to redefine a function to remove a point of discontinuity, and to see the relationships between continuity and differentiability of single variable functions. It has been shown that GeoGebra offers many possibilities which help students to get an intuitive feeling and to visualize an adequate math process. The use of this software's tools allows students to explore a wider range of function types, allows them the flexibility to see mathematical ideas from different perspectives and enables the students to make the connections between symbolic and visual presentations. 


$$
\text { "tmcs-dikovic" — 2009/11/1 — 16:54 — page } 256 \text { — \#16 }
$$

\section{References}

[1] W. Blubaugh, Teaching and Learning Business Calculus Through Technology, Electronic Proceedings of the Seventeenth Annual International Conference on Technology in Collegiate Mathematics New Orleans, Louisiana, 2004, ISBN 0-321-43792-6, Paper S052.

[2] Lj. Dikovich, An Interactive Learning and Teaching of Linear Algebra by Web Technologies: Some Examples, Journal the Teaching of mathematics, The Mathematical Society of Serbia, Beograd (2007), 109-116, ISSN: 1451-4966, Issue: X_2.

[3] E. Dubinsky and K. Schwingendorf, Calculus, Concepts, Computers and Cooperative Learning $\left(C_{4} L\right)$, National Science Foundation funded research and development project, Purdue University, 2004.

[4] Dj. Herceg and D. Herceg, Numerics with GeoGebra in high school, First Centraland Eastern European Conference on Computer Algebra- and Dynamic Geometry Systems in Mathematics Education, University of Pécs, Pollack Mihály Faculty of Engineering, Hungary, 2007.

[5] M. Hohenwarter, J. Hohenwarter, Y. Kreis and Z. Lavicza, Teaching and learning calculus with free dynamic mathematics software GeoGebra, 11th International Congress on Mathematical Education, Monterrey, Nuevo Leon, Mexico, 2008.

[6] M. Hohenwarter and Z. Lavicza, Mathematics teacher development with ICT: towards an International GeoGebra Institute, Vol. 27, no. 3, Proceedings of the British Society for Research into Learning Mathematics, (D. Küchemann, ed.), University of Northampton, UK: BSRLM, 2007.

[7] M. Hohenwarter and J. Preiner, Dynamic Mathematics with GeoGebra, Journal for Online Mathematics and its Applications 7 (2007), Article ID 1448.

[8] M. Hohenwarter, J. Preiner and Yi Taeil, Incorporating GeoGebra into Teaching Mathematics at the College Level, Proceedings of ICTCM 2007, Boston, MA, 2007, http://www.geogebra.org/publications/2007_ICTCM_geogebra/.

[9] W. van Joolingen, Cognitive tools for discovery learning, International Journal of Artificial Intelligence in Education 10 (1999), 385-397.

[10] NCTM, Principles and Standards for School Mathematics, 2000, http://standards.nctm.org.

[11] K. Ruthven, S. Hennessy and S. Brindley, Teacher representations of the successful use of computer-based tools and resources in secondary-school English, mathematics and science, Teaching and Teacher Education 20, no. 3 (2004), 259-275.

[12] H. Waxman, M. Connell and J. Gray, A Quantitative Synthesis of Recent Research on the Effects of Teaching and Learning With Technology on Student Outcomes, NCREL, 2002, www.coe.ufl.edu/Courses/eme5054/Foundations/Articles/waxman.pdf.

[13] O. Wurnig, Some Problem Solving examples of Multiple solutions using cas and dgs, Proceedings of the Discussing Group 9: Promoting Creativity for All Students in Mathematics Education, The 11th International Congress on Mathematical Education, Monterrey, Mexico, 2008. 
"tmcs-dikovic" — 2009/11/1 — 16:54 — page 257 — \#17

[14] http://www.learning-theories.com/discovery-learning-bruner.html.

[15] http://opensource.org/.

[16] http://www.dwheeler.com/oss_fs_why.html.

[17] http://www.gnu.org/philosophy/categories.html.

[18] http://www.geogebra.org.

[19] http://www . math.umn.edu/ rogness/mathlets/xSquaredSin1overX/.

[20] http://www.dean.usma.edu/math/people/Peterson/geogebra/continuity.html.

[21] http://www . math.ucdavis.edu/ kouba.

[22] http://mathdemos.gcsu.edu/mathdemos/piecewise/piecewise_continuity.html.

[23] http://www.geneseocsd.org/webpages/seventhteam/files/AP\%20Calculus\%20January\%2009.doc.

LJUBICA DIKOVIC

BUSINESS TECHNICAL COLLEGE

UZICE

SERBIA

E-mail: dikoviclj@gmail.com

(Received January, 2009) 